\title{
FIBROID MIMICKING SARCOMA
}

\author{
B. Kalpana1, Richa Gupta²
}

${ }^{1}$ HOD, Consultant, Department of Reproductive Medicine, Guru Hospital, Madurai.

2Junior Consultant, Department of Reproductive Medicine, Guru Hospital, Madurai.

HOW TO CITE THIS ARTICLE: Kalpana B, Gupta R. Fibroid mimicking sarcoma. J. Evolution Med. Dent. Sci. 2017;6(74):5326-5327, DOI: $10.14260 /$ Jemds/2017/1156

\section{PRESENTATION OF CASE}

A young 29 years old female came to our clinic with complaints of pain abdomen for last 2 months. There were no menstrual disturbances. She was having one male child. She had gastritis, abdominal bloating and constipation. On general examination, she was mildly pale and vitals were stable. Per abdomen examination revealed a 32 weeks size mass arising from pelvis, soft to firm in consistency and non-tender. There was no evidence of ascites. USG and MRI demonstrated a bulky uterus with anterior wall adenomyosis as shown in Fig. 1. A large necrotic, degenerated fibroid of size $18 \times 16 \mathrm{~cm}$ was seen arising from uterine fundus and posterior wall. It was partly intramural and partly subserosal. The vascularity was mildly increased. Both ovaries were normal. Minimal ascites was evident. Patient's routine blood tests and tumour markers were normal. Patient was planned for surgery.

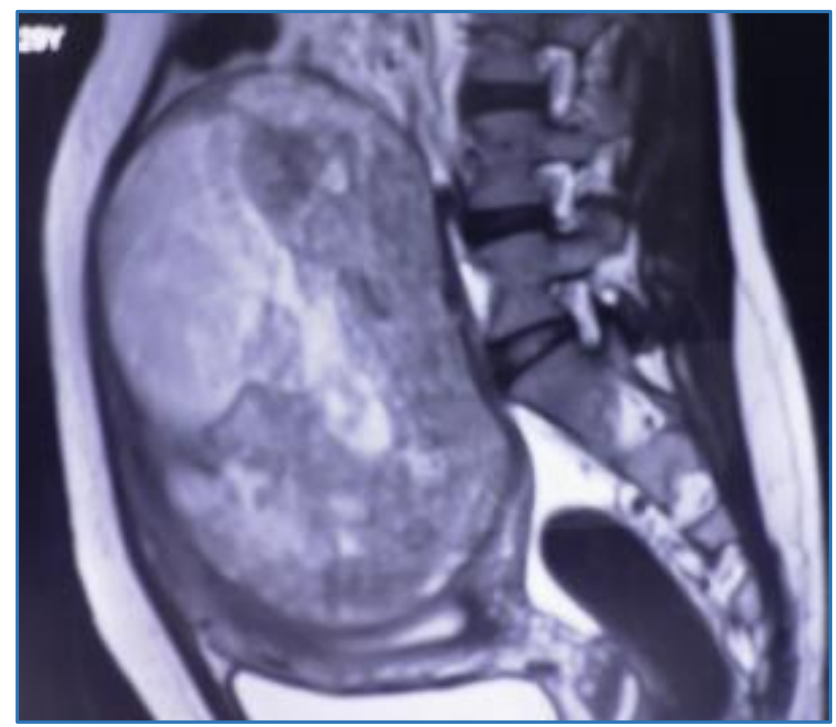

Figure 1. MRI Image showing Abdominal Mass occupying whole Abdominal Cavity

Financial or Other, Competing Interest: None.

Submission 08-08-2017, Peer Review 01-09-2017,

Acceptance 07-09-2017, Published 14-09-2017.

Corresponding Author:

Dr. B. Kalpana

\#4/120 F,

Pandikovil Ring Road,

Madurai-625107,

Tamil Nadu.

E-mail: balamurugan_kalpana@rediffmail.com

DOI: $10.14260 /$ jemds/2017/1156

\section{(c) (i) $($ )}

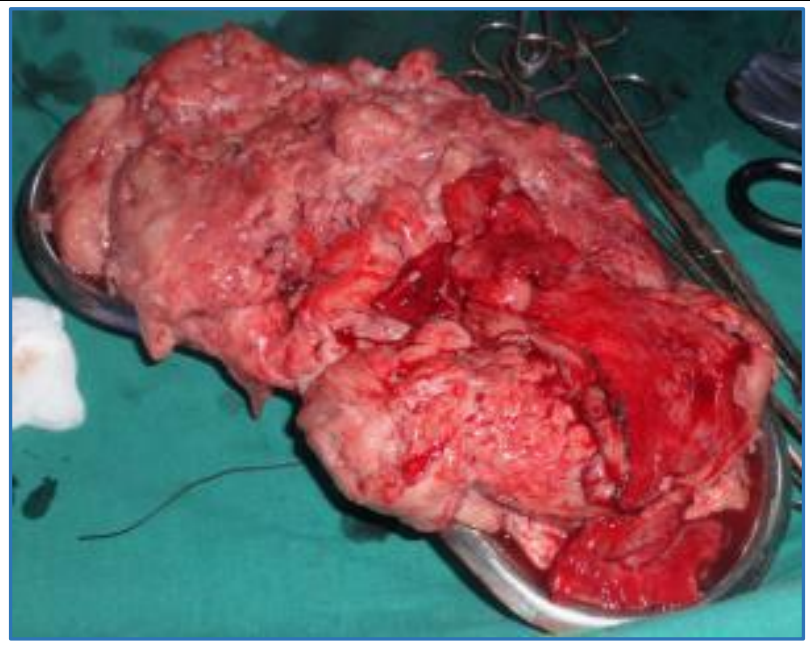

Figure 2. Specimen of Degenerated Fibroid after Surgery

\section{PATHOLOGICAL DISCUSSION}

Laparoscopy was done, which showed a huge mass arising from uterine fundus with soft consistency suggestive of degenerative changes. Decision was taken to convert it to laparotomy, as there was a suspicion of malignancy. Considering patient's age and desire for fertility preservation, conservative surgery was performed after explaining the risk to the husband. Mass was resected and sent for histopathological examination as shown in Figure 2. Uterus was closed in layers.

Excised mass was of $20 \times 16 \mathrm{~cm}$. On cut section it was whitish, firm and multinuclear with areas of cystic degeneration and haemorrhage. Microscopy appearance showed proliferation of smooth muscle fibres arranged in intersecting bundles with areas of hyaline degeneration, cystic changes and haemorrhage. There was no evidence of sarcomatous change. It was a leiomyoma arising from myometrium with degenerative changes.

Post-op course was uneventful and patient was discharged. Patient was asymptomatic during followup. She conceived naturally and delivered a healthy baby at term by LSCS.

\section{DIFFERENTIAL DIAGNOSIS}

Uterine leiomyoma with degenerative changes, Uterine Sarcoma.

\section{DISCUSSION OF MANAGEMENT}

Leiomyomas are the commonest uterine neoplasms occurring in around $20 \%-30 \%$ of women in the reproductive age group.[1-3] Uterine sarcomas are rare and have a poor prognosis. ${ }^{[4]}$ Both present as focal masses in the uterine myometrium. Thus, clinicians who evaluate and manage women with presumed leiomyomas are presented with a 
clinical challenge. Leiomyomas are composed of smooth muscle and fibrous tissue and are benign in nature.[1] Based on their location within the uterine wall, leiomyomas are classified into submucosal/ subendometrial, intramural/ myometrial or subserosal leiomyomas. As leiomyomas enlarge they may outgrow their blood supply, which results in various types of degeneration. These include hyaline, cystic, myxoid or red degeneration and dystrophic calcification. [1,5] Hyalinisation is the most common type of degeneration occurring in $60 \%$ of tumours.[1] Cystic degeneration observed in $4 \%$ of leiomyomas may be considered an extreme sequel of oedema.[1,2] The potential for uterine leiomyomas to grow to an extreme size before causing symptoms is quite remarkable. This is likely due to the relatively large volume of the abdominal cavity, the distensibility of the abdominal wall and the slow growth rate of these tumours.

USG is the primary modality for diagnosing clinically suspected uterine fibroids.[5] USG commonly shows a hypoechoic or heterogeneous uterine mass, whose texture depends on the relative ratio of fibrous tissue to smooth muscle and the presence and type of degeneration.[1] MRI plays a crucial role in determining the origin and nature of a pelvic mass in cases with inconclusive USG features. ${ }^{[6]}$ Typical appearances of leiomyomas are easily recognised on imaging. However, the atypical appearances that follow degenerative changes can cause confusion in diagnosis. Leiomyomas have been misdiagnosed as adenomyosis, haematometra, uterine sarcoma and ovarian masses.

In our case, it was a dilemmatic situation as such a huge leiomyoma with degenerative changes is not so common in a young parous patient. Uterine sarcomas are more common in the elder age group. However, young age does not exclude the diagnosis. Clinical manifestations are not useful to distinguish between leiomyomas and uterine sarcomas, since both typically present with abnormal uterine bleeding, pelvic pain/pressure and a pelvic mass. There is no pelvic imaging modality that can reliably differentiate between benign leiomyomas and uterine sarcomas. Magnetic resonance imaging (MRI) with contrast may be helpful in women in whom there is a suspicion of sarcoma; however, it does not provide a definitive diagnosis.[7] Hence, it is a clinically challenging situation to avoid unnecessary surgery for the purpose of excluding the rare sarcoma and also to offer the option of minimally invasive approaches in patients with leiomyoma, many of whom have not completed their childbearing. We proceeded with conservative surgery to preserve childbearing function and confirmed the diagnosis of a degenerated uterine fibroid.

Although, fibroids typically have a characteristic ultrasound appearance, degenerating fibroids can have variable patterns and pose diagnostic challenges. Ours is an unusual case of a huge leiomyoma with extensive cystic degeneration presenting with abdominal and gastric symptoms, masquerading as uterine sarcoma. We are reporting this case on account of its rarity and diagnostic difficulties.

\section{REFERENCES}

[1] Low SC, Chong CL. A case of cystic leiomyoma mimicking an ovarian malignancy. Ann Acad Med Singapore 2004;33(3):371-4.

[2] Fogata ML, Jain KA. Degenerating cystic uterine fibroid mimics an ovarian cyst in a pregnant patient. J Ultrasound Med 2006;25(5):671-4.

[3] Okizuka H, Sugimura K, Takemori M, et al. MR detection of degenerating uterine leiomyomas. J Comput Assist Tomogr 1993;17(5):760-6.

[4] Brooks SE, Zhan M, Cote $T$, et al. Surveillance, epidemiology, and end results analysis of 2677 cases of uterine sarcoma 1989-1999. Gynecol Oncol 2004;93(1):204-8.

[5] Ahamed KS, Raymond KS. Answer to case of the month \#103 large subserosal uterine leiomyoma with cystic degeneration presenting as an abdominal mass. Can Assoc Radiol J 2005;56(4):245-7.

[6] Karasick S, Toaff ASL, Toaff ME. Imaging of uterine leiomyomas. Am J Roentgenol 1992;158(4):799-805.

[7] Amant F, Coosemans A, Debiec-Rychter M, et al. Clinical management of uterine sarcomas. Lancet Oncol 2009;10(12):1188-98. 